\title{
Haar Wavelet Technique for Solving Fractional Differential Equations with An Application
}

\author{
Mohammed S. Mechee Oday I. Al-Shaher Ghassan A. Al-Juaifri \\ Department of Mathematics, Faculty of Computer Science and Mathematics, \\ Kufa university, Najaf, Iraq \\ mohammeds.abed@uokufa.edu.iq
}

DOI: $10.29304 / j q c m .2019 .11 .1 .461$

\begin{abstract}
:
In this article, the approxmated solutions of ordinary differential equations of fractional order using Haar wavelet and B-spline bases are introduced. The algorithm of collection method is updated using two basis. Several initial value problems has been solved to show the applicability and efficacy of the Haar wavelet and B-spline basis. An application of Lane-Eman equation has been introduced and studied. The approximated results have clearly shown the advantage and the efficiency of the modified method in terms of accuracy and computational time.
\end{abstract}

Keywords Fractional-Order; Ordinary differential equations; Haar Wavelet; Collocation Method; B-spline; Operational matrix Approximated solution. 


\section{Introduction.}

Differential equations (DEs) have an important value in many applications relating to various fields such as engineering, physics, chemistry, biology and economics, however many mathematical models of physical systems are given as DE first-, secondor higher-order. Two types of DEs which depends on the domain of definition of $\mathrm{DE}$ we classified them as complex Des (CDEs) and real DEs (RDEs) each of them classified as follows firstly: Ordinary differential equations (ODEs) which have the branches: delay differential equations (DDEs), fractional differential equations (FrDEs), fuzzy differential equations (FDEs). Secondly: Partial differential equations (PDEs), which have the branches: DDEs, FrDEs and FDEs. Thirdly: Stochastic differential equations which have the branches: DDEs, FrDEs, FDEs. Johy[12]. The ODEs of first- or second order have many applications in branches of mechanical engineering, electrical, civil, chemical and others. The subject of PDEs has a long history with an active contemporary phase. An early phase with a separate focus on string vibrations and heat law through solid bodies. A stimulated of great importance for mathematical analysis to all manner of mathematical, physical and technical problems continues. Such stimulated is a wider concept of functions and integration and the direct relevance of PDEs.

In this paper, Haar wavelet functions used to approximate the solutions of typical ODEs or of fractional-order. Haar wavelets can be written as a family of functions constructed from transformation and dilation of a single function. Haar wavelet transform method is powerful numerical method to use it in solving DEs. Haar wavelet function and its properties are studied and used in solving of the DEs. The useful properties of Haar wavelet transform are studied in solving the DEs. The solutions of them are approximated by the summation of constant multiples of the Haar functions. The other terms of the DE usually found out using some properties of integrating and differentiating . Many researches studied Haar wavelets like Berwal et al. [3] studied the solution of DEs based on Haar operational matrix, Sahoo[23] studied the solution of DEs using Haar wavelet collocation method, Shi et al. (2007) studied the numerical solution of DEs by using Haar wavelets,
Chang \&Piau.[4] used Haar wavelet matrices designation in numerical solution of ODEs, Chen [5] used Haar wavelet approach to ODEs, Li \& Hu [16] solved the fractional Riccati DEs using Haar wavelet while Mechee\&Senu[17] studied the fractional DEs of Lane-Emden type numerically by method of collocation. Shah \& Abbas [24] used Haar wavelet operational matrix method for the numerical solution of fractional order DEs, Saeed\&urRehman[21] used Haar wavelet-quasi linearization technique for fractional nonlinear DEs and Lepik[14] applied Haar wavelet transform to solving IEs and DEs. Weilbeer[29] introduced efficient numerical methods for fractional DEs and their analytical background. Haar wavelet operational matrix and its application for the approximated solution of fractional Bagley Torvik equation has been used by Ray [19], while Shiralashetti et al. [26] used Haar wavelet collocation method for the numerical solution of singular initial value problems. Kilicman \& Al Zhour [13] introduced Kronecker operational matrices for fractional calculus and some applications, Hosseinpour \& Nazemi [10] solved fractional optimal control problems with fixed or free final states by Haar wavelet collocation method, Hsiao [11] constructed Haar wavelet direct method for solving variational problems, Hariharan et al. [8] used Haar wavelet method for solving Fisher's equation, while Aziz \& Amin [2] introduced numerical solution of a class of DDEs and DPDEs via Haar wavelet. Recently, we have studied implementation of different tested problems DEs which are used as mathematical models in many physically applied science and important fields. The approximated solutions of DEs have been derived using Haar wavelet and B-spline basis which shows to be more suitable to approximate the solutions of DE.

\section{Preliminary}

\subsection{Haar Wavelet Functions}

Haar functions have been used since 1910, when they were introduced by Hungarian mathematician (Haar (1910)). The orthogonal set of Haar function is defined as square waves with value of \pm 1 in some interval and zero elsewhere. Then, $h_{0}(x)=1$ during the whole interval $0 \leq x \leq 1$. The second curve $h_{1}(x)$ is the fundamental square wave function which also spans the whole interval $[0 ; 1]$. 
All the other subsequent curve are generated from $h_{1}(x)$ with two operation translation and dilation, $h_{2}(x)$ is obtained from $h_{1}(x)$ with dilation, i.e., $h_{1}(x)$ is compressed from the whole interval $[0 ; 1]$ to half interval $\left[0 ; \frac{1}{2}\right]$ to generate $h_{2}(x), h_{3}(x)$ is the same as $h_{2}(x)$ but shifted to the right direction by $\frac{1}{2}$. Similarly, $h_{2}(x)$ is compressed from the half interval to a quarter interval to generate $h_{4}(x)$. The function $h_{4}(x)$ is translated to the right by $\frac{1}{4}, \frac{2}{4}, \frac{3}{4}$, to generate $h_{5}(x), h_{6}(x)$ and $h_{7}(x)$ respectively. In general, we have the following:

$h_{0}(x)=h_{j}\left(2^{j} x-\frac{k}{2^{j}}\right)$, where $n=2^{j}+k, j \geq 0$, $0<k \leq 2^{j}$.

This orthogonal basis is a reminiscent of the Walsh basis, in which each Walsh function contains many wavelets to fill the interval $[0,1]$ completely, and to form a global basis. While each Haar function contains just one wavelet during some subinterval of time, and remains zero elsewhere the Haar set form a local basis. All the Haar wavelets are orthogonal to each other:

$$
\int_{0}^{1} h_{i}(x) h_{j}(x) d x=2^{-j} \delta_{i j}=\left\{\begin{array}{cc}
2^{-j}, i=j=2^{j+k} \\
0, & i \neq j
\end{array}\right.
$$

However, the functions give a very good transform basis. To obtain a good time resolution for high frequency transients and good frequency resolution for low frequency components, Marled (1982) first introduced the idea of wavelets as a family of functions constructed from translations and dilations of a single function called mother wavelet and defined by

$$
H_{a, b}(t)=\frac{1}{\sqrt{|a|}} H\left(\frac{t-b}{a}\right), a \neq 0, \quad a \in R
$$

where $a$ is scaling parameter measures degree of compression and $\mathrm{b}$ is the translation parameter determines time location of wavelet.

\section{Definition 2.1. (Haar functions )[13]}

The Haar wavelet functions defined as follows on $[0, X]$.

$$
\begin{aligned}
& h_{0}(x)=\frac{1}{\sqrt{M}} \quad, 0 \leq x \leq X \\
& h_{1}(x)=\frac{1}{\sqrt{M}}\left\{\begin{array}{cr}
1, & 0 \leq x \leq \frac{X}{2} \\
-1, & \frac{X}{2} \leq x \leq X \\
0 & , \text { o.w }
\end{array}\right.
\end{aligned}
$$

$$
h_{j}(x)=\frac{1}{\sqrt{M}}\left\{\begin{array}{cc}
\sqrt{2^{j}}, & \frac{k-1}{2^{j}} \leq x \leq \frac{k-\frac{1}{2}}{2^{j}} X \\
-\sqrt{2^{j}}, & \frac{k-\frac{1}{2}}{2^{j}} X \leq x \leq \frac{k}{2^{j}} X \\
0 & , \text { o. } w
\end{array}\right.
$$

For $i=1,2,3, \ldots, m-1, M=2^{j}$ and $i=2^{j}+k-$ 1.

We say that $h_{1}(x)$ is mother function and

$h_{i}(x)=2^{\frac{j}{2}} h_{1}\left(2^{j} x-k\right)$

For $i=1,2,3, \ldots, m-1$

Note that:

$$
\begin{aligned}
\left(h_{p}(x), h_{q}(x)\right) & =\int_{0}^{x} h_{p}(x), h_{q}(x) d x \\
& =\left\{\begin{array}{cc}
\frac{x}{m}, & p \neq q \\
0, & p=q
\end{array}\right.
\end{aligned}
$$

To approximate the function $f(x)$ using Haar functions consider

$$
f(x)=\sum_{i=0}^{m-1} a_{i} h_{i}(x)
$$

$$
\begin{aligned}
& \int_{0}^{x} f(x) h_{j}(x) d x \\
= & \sum_{i=0}^{m-1} a_{i} \int_{0}^{x} h_{i}(x) h_{j}(x) d x, \\
= & a_{j} \int_{0}^{x} h_{j}^{2}(x) d x,
\end{aligned}
$$

Where

\subsection{Spline Functions}

$$
\begin{aligned}
& a_{j}=\frac{\int_{0}^{x} f(x) h_{j}(x) d x}{\int_{0}^{x} h_{j}^{2}(x) d x} \\
= & \frac{m}{X} \int_{0}^{x} f(x) h_{j}(x) d x
\end{aligned}
$$

The spline functions are used in applications of numerical analysis due to they have a wide class of smoothness. One of these applications is data interpolation. The data structure may be either onedimensional or multi-dimensional. In the interpolation, spline functions are normally determined as the minimizers of suitable measures of roughness subject to the interpolation constraints. Smoothness splines may be show as generalizations of interpolation splines where the functions are determined to minimize a weighted linear combination of the average squared approximation error over observed data. 
The spline functions are constructed to be finite dimensional in the applications. Here, we have focus on one-dimensional, polynomial B-splines and use the term B-spline in this restricted sense. The base

$$
\Phi(x)=\left\{\Phi_{1}(x), \Phi_{2}(x), \ldots, \Phi_{n}(x)\right\}
$$

is called $\mathrm{B}$ - spline base of order $\mathrm{n}$ if the basis functions satisfy $\Phi_{i}(x) \in C^{n-1}(\infty,-\infty)$ for $i=$ $1,2, \ldots, n$

First of all, we will partition $[0,1]$ by choosing a positive integer $n$ and defining $h=\frac{1}{n+1}$. This produces the equally-spaced nodes $x_{i}=i h$, for each $i=1,2, \ldots, n+1$. Then, we have defined the basis functions $\left\{\Phi_{i}(x)\right\}_{i=0}^{n+1}$ on the interval $[0,1]$.

\subsubsection{Linear Spline}

The simplest spline is a piecewise polynomial function, with each polynomial having a single variable .The spline $S$ takes values from an interval $[a, b]$ and maps them to $\mathfrak{N}$ where $S:[a, b] \rightarrow$ $\mathfrak{N}$ Since $\mathrm{S}$ is piecewise defined, choose $\mathrm{k}$ subintervals to partition $[a, b]$. The simplest choice of spline functions basis involves piecewise-linear polynomials. The first step is to form a partition of $[0,1]$ by choosing points $x_{0}, x_{1}, \ldots, x_{n}$. Let $h_{i}=$ $x_{i+1}-x_{i}$,for each $i=1,2, \ldots, n$.We have defined the basis functions $\left\{\Phi_{1}(x), \Phi_{2}(x), \ldots, \Phi_{n}(x)\right\}$. Linear spline is linear polynomial $S(x)$ which satisfy $S(x) \in C_{0}(\infty,-\infty)$ To construct linear spline base in which satisfy the boundary conditions $\Phi_{i}(0)=$ $\Phi_{i}(1)$ for $i 1,2, \ldots, n \mathrm{We}$ have constructed the following component linear spline functions:

$$
\Phi_{i}(x)=\left\{\begin{array}{cr}
0 & 0 \leq x \leq x_{i-1} \\
\frac{1}{h_{i-1}}\left(x-x_{i-1}\right), & x_{i-1}<x \leq x_{i} \\
\frac{1}{h_{i}}\left(x_{i+1}-x\right), & x_{i}<x \leq x_{i+1} \\
0, & x_{i+1}<x \leq 1 .
\end{array}\right.
$$

for each $i=1,2, \ldots, n$. We can prove that the functions are orthogonal because $\Phi_{i}(x)$ and $\Phi_{i}^{\prime}(x)$ are nonzero only on $\left(x_{i-1}, x_{i+1}\right)$ such that $\Phi_{i}(x) \Phi_{j}(x)=0$ and $\Phi_{i}^{\prime}(x) \Phi_{j}^{\prime}(x)=0$ if $i \neq$ $j, i-1, j-1$.Consequence $\Phi_{i}(x) \in C(\infty,-\infty)$.

\subsubsection{Quadratic B-Spline}

Quadratic B-spline base is quadratic B-Spline polynomials $S(x)$ which satisfy $S(x) \in C_{0}^{1}(\infty,-\infty)$ To construct quadratic spline base in which satisfy the boundary conditions $\Phi_{i}(0)=\Phi_{i}(1)$ for $\mathrm{i}=1,2, \ldots \mathrm{n}$ We have constructed the following component quadratic spline functions:

$$
\emptyset_{m}(x)=\left\{\begin{array}{cc}
T+3\left(x_{m}-x\right)^{2} ;\left[x_{m-1} ; x_{m}\right] \\
T ; & {\left[x_{m} ; x_{m+1}\right]} \\
& \left(x_{m+2}-x\right)^{2} ; \quad\left[x_{m+1} ; x_{m+3}\right] \\
0 & \text { o.w. }
\end{array}\right.
$$

Where $T=\left(x_{m+2}-x\right)^{2}-3\left(x_{m+1}-x\right)^{2}$

\subsubsection{Cubic B-Spline}

Many researchers used B-cubic spline in numerical analysis. We have defined B cubic spline base as follows:

$$
\begin{gathered}
s(x) \\
=\frac{1}{4}\left\{\begin{array}{c}
0, \quad x<-2 \\
(2+x)^{3}, \quad-2 \leq x \leq-1 \\
(2+x)^{3}-4(1+x)^{3},-1 \leq x \leq 0 \\
(2-x)^{3}-4(1-x)^{3}, 0 \leq x \leq 1 \\
(2-x)^{3}, \quad 1 \leq x \leq 2 \\
0,
\end{array} \quad x>2\right.
\end{gathered}
$$

Consequence $\mathrm{S}(\mathrm{x}) \in \mathrm{C}_{0}^{2}(-\infty, \infty)$. To construct cubic spline base in which satisfy the boundary conditions $\emptyset_{\mathrm{i}}(0)=\emptyset_{\mathrm{i}}(1)$ for $\mathrm{i}=1,2, \ldots, \mathrm{n}$. We have constructed the following component cubic spline functions on the interval $\left[\mathrm{x}_{\mathrm{i}-2}, \mathrm{x}_{\mathrm{i}+2}\right]$ as follows [1]: $\emptyset_{i}(x)$

$$
=\frac{1}{4}\left\{\begin{array}{c}
s\left(\frac{x}{h}\right)-4 s\left(\frac{x+h}{h}\right), \quad i=0 \\
s\left(\frac{x-h}{h}\right)-s\left(\frac{x+h}{h}\right), \quad i=1 \\
s\left(\frac{x-i h}{h}\right), \quad 2 \leq i \leq n \\
s\left(\frac{x-n h}{h}\right)-s\left(\frac{x-(n+2) h}{h}\right), \quad i=n \\
s\left(\frac{x-(n+1) h}{h}\right)-4 s\left(\frac{x-(n+2+h}{h}\right), \quad i=n+1
\end{array}\right.
$$

\subsection{Fractional Derivatives}

The fractional integrals have been defined by many researches as follows:

The left hand Riemann-Liouville fractional derivatives of order $\alpha>0 ; n \in N$ ( $\mathrm{N}$ is natural numbers set), is given by:

$$
D_{x}^{\alpha} f(x)=\frac{1}{\Gamma(n-\alpha)} \frac{d^{n}}{d x^{n}} \int_{0}^{x} \frac{f(t)}{(x-t)^{\alpha-n+1}} d t
$$

\subsubsection{Operational Matrix of the Fractional-Order Integration of the Haar Wavelet}

Shiralashetti \& Deshi [26] had introduced the Haar wavelet operational matrix $F H_{\alpha}$ of integration of the fractional order $\alpha$ is given by 


$$
F H_{\alpha, i}=\left\{\begin{array}{c}
f_{1}, x \in\left[\frac{k}{m}, \frac{k+0.5}{m}\right) \\
f_{2}, x \in\left[\frac{k+0.5}{m}, \frac{k+1}{m}\right) \\
f_{3}, x \in\left[\frac{k+1}{m}, 0\right) \\
0, \quad \text { o.w }
\end{array}\right.
$$

Where

$$
\begin{aligned}
& f_{1}(x)= \frac{1}{\Gamma(n-\alpha)}\left(x-\frac{k}{m}\right)^{\alpha} \\
& f_{2}(x)= \frac{1}{\Gamma(n-\alpha)}\left(\left(x-\frac{k}{m}\right)^{\alpha}-2\left(x-\frac{k+0.5}{m}\right)^{\alpha}\right) \\
& f_{3}(x)=\frac{1}{\Gamma(n-\alpha)}\left(\left(x-\frac{k}{m}\right)^{\alpha}-2\left(x-\frac{k+0.5}{m}\right)^{\alpha}\right. \\
&\left.\quad+\left(x-\frac{k+1}{m}\right)^{\alpha}\right)
\end{aligned}
$$

2.4 Operational Matrix of the Fractional-Order Integration of the B-Spline Bas

\subsubsection{Linear Spline}

We have introduced the linear B-spline operational matrix FSa of integration of the fractional order as follows:

$$
\begin{gathered}
J_{x_{i}}^{\alpha}(x)=\frac{1}{\Gamma(n+2)} \\
0, \quad 0 \leq x \leq x_{i-1} \\
\left\{\begin{array}{c}
\frac{1}{h_{i-1}}\left(x-x_{i-1}\right)^{\alpha+1}, x_{i-1} \leq x \leq x_{i} \\
\frac{1}{h_{i-1}}\left(x-x_{i-1}\right)^{\alpha+1}+\frac{1}{h_{i}}\left(h_{i} \alpha\left(x-x_{i}\right)^{\alpha}-\left(x-x_{i}\right)^{\alpha+1}, x_{i} \leq x \leq x_{i+1}\right. \\
0, x_{i+1} \leq x \leq 1
\end{array}\right.
\end{gathered}
$$

\subsubsection{Quadratic B-Spline}

We have introduced the quadratic B-spline operational matrix $F S_{a}$ of integration of the fractionalorder as follows:

$J_{x}^{\alpha}(x)$

$=\frac{1}{\Gamma(n+3)}\left\{\begin{array}{l}2(x-1)^{\alpha+2}, \\ 2(x-1)^{\alpha+2}-6(x-2)^{\alpha+2}, \\ 2(x-1)^{\alpha+2}-6(x-2)^{\alpha+2}+6(x-3)^{\alpha+2}, \\ 0,\end{array}\right.$

\subsubsection{Cubic B-Spline}

We have introduced the cubic B-spline operational matrix FSa of integration of the fractional order as follows:

$$
J_{x}^{\alpha}(x)=\frac{1}{\Gamma(n+3)}
$$

Mohammed .S/ Oday .I/ Ghassan .A

$$
\left\{\begin{array}{cc}
0, & x<-2 \\
\frac{3}{2} x^{\alpha+3}-2 \leq x \leq-1 & \\
\frac{3}{2} x^{\alpha+3}-6(x-1)^{\alpha+3}, & -1 \leq x \leq 0 \\
\frac{3}{2} x^{\alpha+3}-6(x-1)^{\alpha+3}+9(x-2)^{\alpha+3}, & 0 \leq x \leq 1 \\
\frac{3}{2} x^{\alpha+3}-6(x-1)^{\alpha+3}+9(x-2)^{\alpha+3}-6(x-3)^{\alpha+3}, & 1 \leq x \leq 2 \\
0, & x>2
\end{array}\right.
$$

\section{Analysis of Collection Method[1]}

Define the collocation points $x_{i}=a+i h$ for $i=0,1,2, \ldots, n$ discretize the functions:

$$
\phi(x)=\left\{\phi_{1}(x), \phi_{2}(x), \phi_{3}(x), \ldots \ldots, \phi_{n}(x)\right\}
$$

Suppose

$$
y(x)=\sum_{i=1}^{n} c_{i} \phi_{i}(x)
$$

Put the approximation of $y(x)$ at the point $x_{j}$ in the $\mathrm{DE}$, we get the function coefficient matrix $\Phi_{i, j}(x)=\Phi_{i}\left(x_{j}\right)$ and $\Phi_{i, j}^{\prime}(x)=\Phi_{i}^{\prime}\left(x_{j}\right)$ The matrix of coefficients has the dimension $n \times n$. Any function $y(x)$ which is square integrable in the interval $(0,1)$ can be expressed as an infinite sum of Haar wavelet. The above series terminates at finite terms if $y(x)$ is piecewise constant or can be approximated as piecewise constant during each subinterval [1].

\subsection{The Quadratic B-Spline Base}

Consider the quadratic B-spline Base

$$
s(x)=\left\{s_{1}(x), s_{2}(x), s_{3}(x), \ldots ., s_{n}(x)\right\}
$$

Suppose $y(x)=\sum_{i=1}^{n} c_{i} s_{i}(x)$ The general ODE of $[1,2]$ first-order has the following form:

$[2,3]\left(199_{0}(t) y^{\prime}(t)+a_{1}(t) y(t)=f(t), 0 \leq t \leq 1,(21)\right.$ subject to the initial condition is $y(0)=\alpha$.

\section{Problem 3.1}

$$
y^{\prime}(t)=y(t)=\sin (t)+\cos (t), \quad 0 \leq t \leq 1
$$

subject to the initial condition is $y(0)=0$, The coefficients $\quad$ are $a_{0}(t)=a_{1}(t)=1$ and $f(t)=$ $\sin (t)+\cos (t)$ Consider the quadratic B-spline base, Then, The matrix of coefficients has the following formula:

$$
A_{i j}=s_{i}\left(t_{j}\right)+s_{i}\left(t_{j}\right)
$$


And $\quad b_{i}=\sin t_{i}+\cos t_{i}$ for $\mathrm{i}, \mathrm{j}=1,2, \ldots, \mathrm{n}$. By solving the system of coefficients $A c=b$ we will obtain the coefficients of approximation where $\mathrm{c}=[.1, .2,-.1, .001,4]$.

\subsection{The Haar Wavelet Base}

We introduce the Haar wavelet technique for solving general linear first-order ODEs.

\subsubsection{First-Order Linear First-Order ODEs}

Consider the following general linear first-order ODE:

$$
\begin{aligned}
& y^{\prime}(t)+f(t) y(t)=g(t), 0 \leq t \leq a, f(t) \neq 0 \\
& y(0)=\beta
\end{aligned}
$$

Substituting $t=a x$ in Equation (23) which reduces to $y^{\prime}(x)+a f(a x) y(a x)=a g(a x), 0 \leq x \leq$

$a, f(x) \neq 0$,

$$
y(0)=\beta \text {. }
$$

We assume that $y^{\prime}(x)=\sum_{i=1}^{k} c_{i} h_{i}(x)$

where $c_{i} s$ are Haar coefficients to be determined. Integrating Equation (27) with respect to $x$, we get the following

$y(x)=\beta+\sum_{i=1}^{k} c_{i} p_{1, i}(x)$

Substituting Equations (27) and (28) in Equation (25), we get the following system of equation:

$\sum_{i=1}^{k} c_{i} h_{i}(x)+a f(x)\left(\beta+\sum_{i=1}^{k} c_{i} p_{1, i}(x)\right)=a g(x)$ (29)

Put $x=t_{j}$ for $j=1,2, \ldots n$. in Equation (29), we get linear system in which the matrix of coefficients has the following formula:

$A_{i j}=\left(1+a f\left(t_{i}\right)\right) h_{i}\left(t_{i}\right) \quad$ and $b_{i}=a g\left(t_{i}\right)$

for $j, i=1,2, \ldots n$ By solving the linear system of coefficients $A c=b$ we obtain the coefficients of approximated solution.

\subsection{Fractional Differential equations with Haar Base}

We will introduce the Haar wavelet technique for solving FrDEs

Problem 3.2. Consider the general fractional-order linear DE

$$
\begin{aligned}
& y^{\alpha}(t)+A(t)+B(t) y(t)=C(t) \\
& 0 \leq t \leq a ; n-1<\alpha<n
\end{aligned}
$$

subject to initial conditions $y_{j}(0)=a_{j}$ for $j=0,1,2, \ldots, n-1$ where $A(t), B(t)$ and $C(t)$ are given functions,$a_{j} s$ are arbitrary constants and $\alpha$ is a parameter describing the order of the fractional derivative. The general response expression contains a parameter describing the order of the fractional derivative that can be varied to obtain various responses. Substituting $t=a x$ in Equation (27) which reduces to

$$
\begin{gathered}
y^{\alpha}(a x)+a A(a x)+a B(a x) y(a x)=a C(a x) \\
0 \leq x \leq 1 ; n-1<\alpha<n \\
y_{j}(0)=a_{j}
\end{gathered}
$$

We assume that $y^{\alpha}(x)=\sum_{i=1}^{k} c_{i} h_{i}(x)$

If $\alpha=\frac{1}{2}$,integrating Equation (28) once, we get

$y(x)=a_{0}+\sum_{i=1}^{k} c_{i} F H_{\frac{1}{2}, i}(x)$

Substituting Equations (32) and (33) in Equation (31), we get

$$
\begin{aligned}
\sum_{i=1}^{k} c_{i} h_{i}(x)-a A(x) & -a B(x)\left(a_{0}+\sum_{i=1}^{k} c_{i} F H_{\frac{1}{2}, i}(x)\right) \\
= & a C(x)
\end{aligned}
$$

If $\alpha=\frac{3}{2}$,integrating Equation (28) once, we get $y^{\frac{1}{2}}(x)=a_{1}+\sum_{i=1}^{k} c_{i} F H_{\frac{1}{2}, i}(x)$

And

$y(x)=a_{0}+a_{1} x+\sum_{i=1}^{k} c_{i} F H_{\frac{3}{2}, i}(x)$

Substituting Equations (32) and (35) in Equation (31), we get

$$
\begin{aligned}
& \sum_{i=1}^{k} c_{i} h_{i}(x)-a A(x)-a B(x)\left(a_{0}+a_{1} x+\right. \\
& \left.\sum_{i=1}^{k} c_{i} F H_{\frac{3}{2}, i}(x)\right)=a C(x)
\end{aligned}
$$

Put $x=t_{j}$ for $j=1,2, \ldots, n$. in Equation (35) in case $\alpha=\frac{1}{2}$, or in Equation (35) in case $\alpha=\frac{3}{2}$,we get the linear system in which the matrix of coefficients has the following formula:

and

$$
\boldsymbol{A}_{\boldsymbol{i j}}=\boldsymbol{h}_{\boldsymbol{i}}\left(\boldsymbol{t}_{\boldsymbol{j}}\right)+\boldsymbol{a} \boldsymbol{B}\left(\boldsymbol{t}_{\boldsymbol{j}}\right) F H_{\alpha, i}\left(\boldsymbol{t}_{\boldsymbol{j}}\right)
$$

$$
b_{i}=c\left(\boldsymbol{t}_{\boldsymbol{j}}\right)+a A\left(\boldsymbol{t}_{\boldsymbol{j}}\right)-a a_{0} B\left(\boldsymbol{t}_{\boldsymbol{j}}\right)
$$

for $i, j=1,2, \ldots, n$. By solving the linear system of coefficients, we obtain the coefficients of approximated solution $y(t)$ of Equation (31).

\subsection{Fractional Differential equations with B- \\ Spline Base}

We will introduce the B-spline technique for solving FrDE (31). Consider the quadratic B-spline base

$$
s(x)=\left\{s_{1}(x), s_{2}(x), s_{3}(x), \ldots ., s_{n}(x)\right\}
$$

Suppose $y(x)=\sum_{i=1}^{n} c_{i} s_{i}(x)$

We assume that $y^{, \alpha}(x)=\sum_{i=1}^{k} c_{i} s_{i}(x)$ 
If $\alpha=\frac{1}{2}$,integrating Equation (37) once, we get

$y(x)=a_{0}+\sum_{i=1}^{k} c_{i} F S_{\frac{1}{2}, i}(x)$

Substituting Equations (37) and (38) in Equation (31), we get

$$
\begin{aligned}
\sum_{i=1}^{k} c_{i} S_{i}(x)-a A(x) & -a B(x)\left(a_{0}+\sum_{i=1}^{k} c_{i} F S_{\frac{1}{2}, i}(x)\right) \\
= & a C(x)
\end{aligned}
$$

If $\alpha=\frac{3}{2}$,integrating Equation (31) once, we get

$y(x)=a_{0}+a_{1} x+\sum_{i=1}^{k} c_{i} F S_{\frac{3}{2}, i}(x)$

Substituting Equations (32) and (39) in Equation (31), we get

$\sum_{i=1}^{k} c_{i} S_{i}(x)-a A(x)-a B(x)\left(a_{0}+a_{1} x+\right.$

$\left.\sum_{i=1}^{k} c_{i} F S_{\frac{3}{2}, i}(x)\right)=a C(x)$

Put $x=t_{j}$ for $j=1,2, \ldots, n$. in Equation (39) in case $\alpha=\frac{1}{2}$, or in Equation (35) in case $\alpha=\frac{3}{2}$,we get the linear system in which the matrix of coefficients has the following formula:

$$
\boldsymbol{A}_{\boldsymbol{i} \boldsymbol{j}}=\boldsymbol{h}_{\boldsymbol{i}}\left(\boldsymbol{t}_{\boldsymbol{j}}\right)+\boldsymbol{a} \boldsymbol{B}\left(\boldsymbol{t}_{\boldsymbol{j}}\right) F S_{\alpha, i}\left(\boldsymbol{t}_{\boldsymbol{j}}\right)
$$

and

$$
b_{i}=c\left(\boldsymbol{t}_{\boldsymbol{j}}\right)+a A\left(\boldsymbol{t}_{\boldsymbol{j}}\right)-a a_{0} B\left(\boldsymbol{t}_{\boldsymbol{j}}\right)
$$

for $i, j=1,2, \ldots, n$. By solving the linear system of coefficients, we obtain the coefficients of approximated solution $y(t)$ of Equation (31).

\section{Lane-Emden Fractional Differential Equation}

We generalize the definition of Lane- Emden equations up to fractional order as following:

$$
D^{\alpha} y(t)+\frac{k}{t^{\alpha-\beta}} D^{\beta} y(t)+f(t, y)=g(t)
$$

$0<t \leq 1, k>0$.

with the initial condition $y(0)=A ; y_{0}(0)=B$ where $1<\alpha \leq 2 ; 0<\beta \leq 1$ and $A ; B$ are constants

and $f(t ; y)$ is a continuous real-valued function and $g(t ; y) \in[0,1]$ : The theory of singular boundary value problems has become an important area of investigation in the past three decades. One of the equations describing this type is the Lane-Emden equation. Lane-Emden type equations, first published by Homer Lane (1870), and further explored in detail by Emden [6], represents such phenomena and having significant applications, is a second-order ODE with an arbitrary index, known as the polytropic index, involved in one of its terms.
The Lane-Emden equation describes a variety of phenomena in physics and astrophysics. Mechee\&Senu[18] imposed the Lane-Emden DE of fractional order and the approximate solution is obtained by employing the method of power series and a numerical solution is established by the least squares method for these equations. Mechee\&Senu[17] approximate the solution of DE by employing the method of power series and the numerical solution is established by collection method.

\section{Analysis of the Method of Solution Lane- Emden of Fractional Order}

Berwal et al. [3] studied the solution of DEs based on Haar operational matrix, Sahoo[23] studied the solution of DEs using Haar wavelet collocation method, Shi et al. [25] studied the 10numerical solution of DEs by using Haar wavelets, Chen [5] used Haar wavelet approach toODEs, Li \& Hu [16] solved the fractional Riccati DEs using Haar wavelet while Saeedi et al. [22] introduced an operational Haar wavelet method for solving fractional Volterra integral equations, Lepik[15] solved fractional integral equations by the Haar wavelet method, Saeed $\&$ Rehman[20] used Haar wavelet-quasi linearization technique for fractional nonlinear DEs,Lepik[15] solved the fractional integral equations by the Haar wavelet method, Wang et al. [28] used Haar wavelet method for solving fractional PDEs numerically. In Equation (40), consider $\alpha>b, f(t, y)=\frac{1}{t^{\alpha+2}} y(t)$ and $g(t)=0 \quad$ However, $\quad D^{\alpha} W(t)=a h(t)=$ $\sum_{i=1}^{m} c_{i} h_{i}(x)$ and

$$
\begin{gathered}
, D^{\alpha} W(t)=\left(I^{\alpha-\beta} D^{\alpha}\right) W(t)+W^{\beta}(0) \\
=a P^{\alpha-\beta} h(t)+W^{\beta}(0) \\
W(t)=\left(I^{\alpha} D^{\alpha}\right) W(t)+W(0) \\
=a P^{\alpha} h(t)+A
\end{gathered}
$$

Hence,

$$
\begin{gathered}
a h(t)+\frac{k}{t^{\alpha-\beta}} a p^{\alpha-\beta} h(t)+W^{\beta}(0)+a p^{\alpha} h(t)+A \\
=\operatorname{ch}(t)
\end{gathered}
$$

If we consider $\alpha=\frac{3}{2}$ and $\beta=\frac{1}{2}$ we solve the system of equations to obtainthe coefficients $\left(c_{0}, c_{1}, c_{2}, \ldots, c_{m}\right)$. 
Mohammed .S/ Oday .I/ Ghassan .A

\section{Comparison Study Using Numerical Collection Method}

Collocation method for solving DEs is one of the most powerful approximated methods. This method has its basis upon approximate the solution of FrDEs by a series of complete sequence of functions, a sequence of linearly independent functions which has no non-zero function perpendicular to this sequence of functions. In general, $y(t)$ is approximated by Mechee\&Senu [17]

$y(x)=\sum_{i=1}^{n} a_{i} \theta_{i}(x)$

where $a_{i}$ for $i=0,1,2, \ldots, n$ are an arbitrary constants to be evaluated and $\theta_{i}$ for $i=$ $0,1,2, \ldots$, naregiven set of functions. Therefore, the problem in Equation (40) of evaluating $y(t)$ is approximated by (42) then, is reduced to the problem of evaluating the coefficients for . $i=$ $0,1,2, \ldots, n$ Let $\left\{t_{1}, t_{2}, \ldots, t_{n}\right\}$ is a partition to interval $[0,1]$ and $t_{j}=j h$ and $h=\frac{1}{n} n$ and $j=0,1,2, \ldots, n$ See the comparison of absolute errors of the problem using numerical collection method with polynomial basis and Haar wavelet basis.

\section{Discussion and Conclusion}

The numerical solutions of ordinary differential equations of fractional order using Haar wavelet and B-spline bases have been studied. Haar wavelet technique is used to approximate the solution of the differential equations. The algorithm of collection method is updated for using the two basis. An application of Lane-Emden has been studied numerically. The numerical results have clearly shown the advantage and the efficiency of the modified method in terms of accuracy and computational time.

\section{Conflict of Interests}

The authors declare that there is no conflict of interests regarding the publication of this paper. Table 1: Absolute Errors of Example1 Using Numerical Collection Method with (a) Polynomial Basis (b) Haar wavelet Basis

\begin{tabular}{lccccc}
\hline$n \backslash t$ & 0 & 0.25 & 0.5 & 0.75 & 1 \\
\hline 5 & 0 & $1.3345 \mathrm{e}-3$ & 0.0015 & $5.0673 \mathrm{e}-3$ & $3.6339 \mathrm{e}-3$ \\
& 0 & $1.311 \mathrm{e}-3$ & 0.0005 & $5.0683 \mathrm{e}-3$ & $3.6229 \mathrm{e}-3$ \\
\multirow{2}{*}{10} & 0 & $1.3232 \mathrm{e}-5$ & $2.6342 \mathrm{e}-5$ & $1.5634 \mathrm{e}-6$ & $4.1443 \mathrm{e}-5$ \\
& 0 & $1.3211 \mathrm{e}-5$ & $2.1212 \mathrm{e}-5$ & $1.2341 \mathrm{e}-6$ & $4.0101 \mathrm{e}-5$ \\
\multirow{2}{*}{50} & 0 & $2.3416 \mathrm{e}-7$ & $1.6611 \mathrm{e}-7$ & $5.1126 \mathrm{e}-7$ & $2.1233 \mathrm{e}-7$ \\
& 0 & $2.1414 \mathrm{e}-7$ & $1.2211 \mathrm{e}-7$ & $5.2233 \mathrm{e}-7$ & $2.1266 \mathrm{e}-7$ \\
100 & 0 & $4.9383 \mathrm{e}-8$ & $3.4453 \mathrm{e}-8$ & $5.0347 \mathrm{e}-8$ & $6.4332 \mathrm{e}-7$ \\
& 0 & $4.9121 \mathrm{e}-8$ & $3.4564 \mathrm{e}-8$ & $5.0111 \mathrm{e}-8$ & $6.4222 \mathrm{e}-7$ \\
\hline
\end{tabular}

\section{References}

[1] J. D. Faires and R. Burden, Numerical Methods, Pacific Grove, 2003.

[2] I. Aziz and R. Amin, Numerical solution of a class of delay differential and delay partial differential equations via Haar wavelet, Applied Mathematical Modelling, 2016.

[3] N. Berwal, D. Panchal, and C. Parihar, Solution of differential equations based on Haar operational matrix, Palestine journal of mathematics, 2014.

[4] P. Chang and P. Piau, Haar wavelet matrices designation in numerical solution of ordinary differential equations, International Journal of Applied Mathematics, 2008.

[5] R. Chen, Haar Wavelet Approach To Ordinary Differential Equation, California State Polytechnic University, Pomona, 2016.

[6] R. Emden, Anwendungen der mechanischen Warmentheorie auf Kosmologie und metheorologische Probleme, Berlin, 1907.

[7] A. Haar, Zur theorie der orthogonalen funktionensysteme, Mathematische Annalen, 1910.

[8] G. Hariharan, K. Kannan and K. Sharma, Haar wavelet method for solving Fisher's equation, Applied mathematics and computation, 2009.

[9] J. H. Lane, On the theoretical temperature of the Sun under the hypothesis of a gaseous mass maintaining its volume by internal heat and depending on the laws of gases known to terrestrial experiment, American Journal of Science, 1870 
[10] S. Hosseinpour and A. Nazemi, Solving fractional optimal control problems with fixed or free final states by Haar wavelet collocation method, IMA Journal of Mathematical Control and Information, 2015.

[11] C.-H Hsiao, Haar wavelet direct method for solving variational problems, Mathematics and computers in simulation, 2004.

[12] A. K Johy, A Study of Differential Equations in Complex Domain, Thesis, university of Kufa 2017.

[13] A. Kilicman and Z. A. A. Al Zhour, Kronecker operational matrices for fractional calculus and some applications, Applied Mathematics and Computation, 2007.

[14] ] Ü. Lepik, Application of the Haar wavelet transform to solving integral and differential equations, Proceedings of the Estonian Academy of Sciences, 2007.

[15] Ü. Lepik, Solving fractional integral equations by the Haar wavelet method, Applied Mathematics and Computation, 2009.

[16] Y.-1 Li and L. Hu, Solving fractional Riccati differential equations using Haar wavelet, Third International Conference on Information and Computing, 2010.

[17] M. S. Mechee, and N. Senu , Numerical study of fractional differential equations of lane emden type by method of collocation, Applied Mathematics, 2012.

[18] M. S. Mechee and N. Senu, Numerical study of fractional differential equations of lane-emden type by the least square method, International Journal of Differential Equations and Applications, 2012.
[19] S. S. Ray, On Haar wavelet operational matrix of general order and its application for the numerical solution of fractional Bagley Torvik equation, Applied Mathematics and Computation, 2012.

[20] U. Saeed and M. Rehman, Haar wavelet Picard method for fractional nonlinear partial differential equations, Applied Mathematics and Computation, 2015.

[21] U. Saeed and M. Rehman, Haar waveletquasilinearization technique for fractional nonlinear differential equations, Applied Mathematics and Computation, 2013.

[22] H. Saeedi, Mollahasani, N. Moghadam, and G. N. Chuev, An operational Haar wavelet method for solving fractional Volterra integral equations, International Journal of Applied Mathematics and Computer Science, 2011.

[23] B. Sahoo and S. S. Ray, A study on solution of differential equations using Haar wavelet collocation method, National Insttute of Technology Rourkela, 2013.

[24] F. A. Shah and R. Abbas, Haar wavelet operational matrix method for the numerical solution of fractional order differential equations, Nonlinear Engineering, 2012.

[25] Z. Shi, L.-Y. Deng and Q.-J Chen, Numerical solution of differential equations by using Haar wavelets, International Conference on Wavelet Analysis and Pattern Recognition, 2007.

[26] S. Shiralashetti, and A. Deshi, Haar Wavelet Collocation Method for so solving Riccati and Fractional Riccati Differential 
Mohammed .S/ Oday .I/ Ghassan .A

\title{
مويجات هار لحل المعادلات التفاضلية الكسورية مع تطبيقات
}

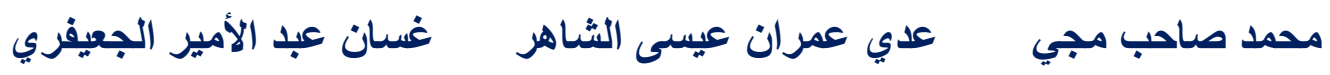 \\ كلية علوم الحاسوب والرياضيات-جامعة الكوفة
}

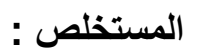

قدمت في هذا البحث الحلول العددية للمعادلات التفاضلية الاعتيادية ذات الرتب الكسرية باستخدام أساس مويجات هار و أساس بي سبلاين. طورت طريقة الحشد باستخدام الأساسين. نم حل بعض مسائل القيم الأبتدائية لتبين كفاءة مويجات

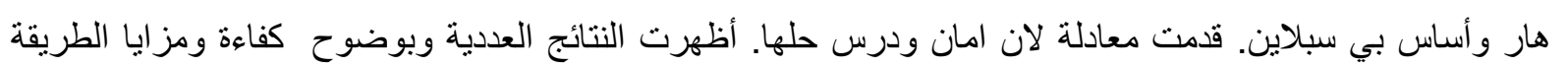
المحورة بالدقة والوقت الأحتسابي. 